Research Paper

\title{
CHD4 as an important mediator in regulating the malignant behaviors of colorectal cancer
}

\author{
Chia-Lo Chang ${ }^{1}$, Chi-Ruei Huang 2,3, Shu-Jyuan Chang4,5, Chun-Chieh Wu ${ }^{4}$, Hong-Hwa Chen ${ }^{1}$, Chi-Wen Luo ${ }^{6,7 凶}$ \\ and Hon-Kan Yip $2,3,8,9,10,11 \bowtie$
}

1. Division of Colorectal Surgery, Department of Surgery, Kaohsiung Chang Gung Memorial Hospital and Chang Gung University College of Medicine, Kaohsiung, Taiwan.

2. Center for Shockwave Medicine and Tissue Engineering, Kaohsiung Chang Gung Memorial Hospital, Kaohsiung, Taiwan.

3. Division of Cardiology, Department of Internal Medicine, Kaohsiung Chang Gung Memorial Hospital and Chang Gung University College of Medicine, Kaohsiung, Taiwan.

4. Department of Pathology, Kaohsiung Medical University Hospital, Kaohsiung, Taiwan.

5. Department of Pathology, College of Medicine, Kaohsiung Medical University, Kaohsiung, Taiwan.

6. Department of Surgery, Kaohsiung Medical University Hospital, Kaohsiung, Taiwan.

7. Division of Breast Surgery, Department of Surgery, Kaohsiung Medical University Hospital, Kaohsiung, Taiwan.

8. Institute for Translational Research in Biomedicine, Kaohsiung Chang Gung Memorial Hospital Kaohsiung, Taiwan.

9. Department of Medical Research, China Medical University Hospital, China Medical University, Taichung, Taiwan.

10. Department of Nursing, Asia University Taichung, Taiwan.

11. Division of Cardiology, Department of Internal Medicine, Xiamen Chang Gung Hospital, Xiamen, Fujian, China.

$\triangle$ Corresponding author: Chi-Wen Luo, Department of Surgery, Kaohsiung Medical University Hospital, 100 Tzyou 1st Road, Kaohsiung city 80756, Taiwan. Tel.: +886-7-312-1101 (ext2260); Fax: +886-7-316-5011; E-mail: 1070598@kmuh.org.tw; Hon-Kan Yip, Division of Cardiology, Department of Internal Medicine, Kaohsiung Chang Gung Memorial Hospital, Kaohsiung city 83301, Taiwan. Tel.: +886-7-7317123; Fax: +886-7-7322402; E-mail: han.gung@msa.hinet.net.

(c) The author(s). This is an open access article distributed under the terms of the Creative Commons Attribution License (https://creativecommons.org/licenses/by/4.0/). See http://ivyspring.com/terms for full terms and conditions.

Received: 2020.12.09; Accepted: 2021.03.28; Published: 2021.04.12

\begin{abstract}
Colorectal cancer (CRC) has ranked first in terms of incidence in Taiwan. Surgical resection combined with chemo-, radio-, or targeted-therapies are the main treatments for CRC patients in current clinical practice. However, many CRC patients still respond poorly to these treatments, leading to tumor recurrence and an unacceptably high incidence of metastasis and death. Therefore, appropriate diagnosis, treatment, and drug selection are pressing issues in clinical practice.

The Mi-2/nucleosome remodeling and deacetylase complex is an important epigenetic regulator of chromatin structure and gene expression. An important component of this complex is chromodomainhelicase-DNA-binding protein 4 (CHD4), which is involved in DNA repair after injury. Recent studies have indicated that CHD4 has oncogenic functions that inhibit multiple tumor suppressor genes through epigenetic regulation. However, the role of CHD4 in CRC has not yet been well investigated.

In this study, we compared CHD4 expression in CRC patients from The Cancer Genome Atlas database. We found higher levels of CHD4 expression in CRC patients. In a series of in vitro experiments, we found that CHD4 affected cell motility and drug sensitivity in CRC cells. In animal models, the depletion of CHD4 affected CRC tumor growth, and the combination of a histone deacetylase 1 (HDACl) inhibitor and platinum drugs inhibited CHD4 expression and increased the cytotoxicity of platinum drugs. Moreover, CHD4 expression was also a prognostic biomarker in CRC patients.

Based on the above results, we believe that CHD4 expression is a viable biomarker for predicting metastasis CRC patients, and it has the potential to become a target for drug development.
\end{abstract}

Key words: colorectal cancer, $\mathrm{CHD} 4$, metastasis, drug resistance

\section{Introduction}

Colorectal cancer (CRC) represents $15 \%$ of all cancers worldwide [1]. CRC is a multistep disorder caused by the accumulation of genetic and epigenetic aberrations under microenvironmental influence. Gene abnormalities, such as those in $p 53, W N T$, DNA mismatch repair genes, and RAS, have been considered to drive the development of a benign adenoma to a carcinoma or metastatic disease $[2,3]$. Previous studies have suggested that heritable genetic changes and genomic instability also played 
important roles in CRC development. In addition, chronic inflammation was considered a risk factor for the CRC development and metastasis [4]. CRC occurrence have also been linked to several environmental factors [5]. In recent years, epigenetic mechanisms were found to record the effects of environmental challenges on the genome level. Therefore, they play important roles in the pathogenesis of inflammation-associated CRC [6]. Despite advancements in drug development and diagnostic methods, CRC is still the third leading cause of cancer-related deaths worldwide [7]. Thus, increasing the survival rate of CRC remains a major challenge for medical institutions worldwide.

Many investigators have studied epigenetic alterations in the CRC cell genome and cancer development over the last two decades [8-11]. Epigenetic aberrations play important roles in affecting every aspect of tumor development process from tumor initiation to metastasis [3]. Although multiple epigenetic mechanisms are involved in the pathogenesis of different cancer types and crossinteract with one other, DNA methylation and histone modifications in the gene promoter regions are the most extensively studied mechanisms. These were also suggested to be the main mediators of CRC epigenetic inheritance in cancer cells $[12,13]$. Ongoing studies have focused on the potential role of recently discovered epigenetic regulators in the treatment of cancer patients and their potential prognostic and predictive values [14].

The nucleosome remodeling and deacetylase (NuRD) complex has been suggested to play important roles in regulating chromatin remodeling, gene expression, and cell cycle progression in normal development and tumorigenesis [15-17]. Among the components of the NuRD complex, chromodomain helicase DNA binding protein 4 (CHD4) is the largest subunit. It is involved in the regulation of cell cycle and DNA repair [18-20]. A prior study also suggested that CHD4 controlled homologous recombination repair to maintain genome stability. Another study indicated that CHD4 could initiate and maintain the epigenetic suppression of multiple tumor suppressor genes, implying that $\mathrm{CHD} 4$ has oncogenic functions [21]. CHD4 deficiency has potential therapeutic implications in tumors [22]. Knockdown of CHD4 could overcome the resistance of approved drugs in acute myeloid leukemia cells or in human epidermal growth factor receptor 2-positive patients [23, 24] and also inhibit tumor growth and increase cytotoxicity in breast cancer [24-27]. Together with CHD4, histone deacetylase 1 (HDAC1) and HDAC2 are the core subunits of the NuRD complex. These interact to maintain the silencing of tumor suppressor genes
(TSGs). Depletion of CHD4 is synergistic with DNA methyltransferase inhibition in reducing cancer cell viability. In relation to the reactivation of tumor suppressor genes, the combined inhibition of these proteins may be beneficial for cancer treatment [28]. However, another study indicated that CHD4 was a pan-cancer biomarker to another HDAC inhibitor sensitivity in colon and breast cancer cells [29]. Therefore, it is necessary to understand the role of CHD4 in regulating the malignant characteristics of CRC.

In this study, we aimed to clarify the role of CHD4 in CRC, to explore the feasibility of using $\mathrm{CHD} 4$ as a prognostic biomarker in CRC patients, and to elucidate whether CHD4 could be a target for future anticancer drug development for CRC patients.

\section{Materials and Methods}

\section{Cell lines}

Colorectal cancer cell lines DLD-1, HCT-116, and HT-29 (ATCC, Manassas, VA, USA) were used in this study. Cells were maintained in Dulbecco's modified Eagle's medium (DMEM) supplemented with 10\% fetal bovine serum (FBS) (Hyclone Laboratories Inc., South Logan, UT, USA) and antibiotics at $37^{\circ} \mathrm{C}$ in a humidified atmosphere with $5 \% \mathrm{CO}_{2}$.

\section{Antibodies}

Antibodies against CHD4 (GTX124186) were purchased from GeneTex Inc. (Hsinchu City, Taiwan, R.O.C). Antibodies against Caspase 3 (\#9662S), cleaved poly (ADP-ribose) polymerase (PARP) (\#9541), Vimentin (\#5741), N-cadherin (\#5296), E-cadherin (\#13116), matrix metalloproteinase

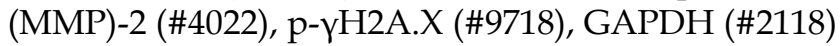
and Actin (\#8480) were purchased from Cell Signaling Technology (Beverly, MA, USA). Mouse (GTX213111) and rabbit (GTX213110) IgG (HRP) antibodies from GeneTex Inc. were used as the secondary antibodies.

\section{Immunoblotting}

Protein extraction and immunoblotting were performed as previously described [30,31]. In brief, cells were lysed in the protein extraction buffer (Thermo Scientific, Rockford, IL, USA). Then, the proteins were collected, loaded on a sodium dodecyl sulfate-polyacrylamide gel, and transferred to nitrocellulose. Immunoblotting was performed using primary and secondary antibodies.

\section{CHD4 short interfering RNA transfection}

Short interfering RNA (siRNA) for human CHD4 (L-009774-00-0005) and negative control (CN-00100001-05) were purchased from Dharmacon (Dharmacon Life Technologies, Cologne, Germany). Appropriate 
non-targeting and specific siRNA were transfected to cells following to the manufacturer's protocol (Thermo, Carlsbad, CA, USA).

\section{CHD4 shRNA clone transfection}

shRNA clones were obtained from the National RNAi Core Facility Platform from Academia Sinica, Taiwan. Individual clones were identified by their unique TRC number (e.g., shCHD4: TRCN0000021363, shLacZ: TRCN0000072233). Transient transfection of cells with plasmids was performed following the manufacture's protocol. Transfected cells were selected using puromycin, and the efficiency of CHD4 silencing was evaluated using real-time reverse transcription-polymerase chain reaction.

\section{Cell proliferation assay}

Cells $\left(1 \times 10^{4}\right)$ were seeded in each well of a 24-well plate. After treatment with different doses of platinum drugs for at least $72 \mathrm{~h}$, the cells were stained with 3-(4,5-dimethylthiazol-2-yl)-2,5-diphenyltetrazolium bromide (MTT) solution (Sigma, St. Louis, MO, USA) and incubated for $1.5 \mathrm{~h}$. Formazan crystals were solubilized in dimethyl sulfoxide (Sigma, St. Louis, MO, USA) and measured at $590 \mathrm{~nm}$. In addition, the apoptotic cell death was evaluated using staining with FITC Annexin V apoptosis detection kit (BD Biosciences, San Jose, CA, USA) and detected using flow cytometry (LSR II Flow Cytometer, BD Biosciences). Each experiment was repeated at least three times.

\section{Wound healing assay}

Cells were seeded in two-well silicone inserts (ibidi GmBH, Planegg, Germany) and cultured in the culture medium at $37^{\circ} \mathrm{C}$ in a $5 \% \mathrm{CO}_{2}$ incubator for 24 $h$. Then, the inserts were removed. Images were then captured at 0 to $48 \mathrm{~h}$. Each experiment was repeated at least three times.

\section{Transwell cell invasion assay}

Cell invasion assays were performed as previously described [32]. Cells were seeded in the upper insert chamber which pre-coated with Matrigel and containing serum-free medium. A culture medium was added to the bottom chamber. After $24 \mathrm{~h}$ of incubation period, cells were rinsed and stained with Giemsa solution (Sigma, St. Louis, MO, USA), and the number of invading cells was counted. Each experiment was repeated at least three times.

\section{Ethics statement}

Animal experimental procedures were approved by the Institute of Animal Care and Use Committee at Kaohsiung Chang Gung Memorial Hospital (Affidavit of Approval of Animal Use Protocol No. 2017110301) and performed in accordance with the Guide for the Care and Use of Laboratory Animals. Animals were housed in an Association for Assessment and Accreditation of Laboratory Animal Care International (Frederick, MD, USA)-approved animal facility in Kaohsiung Chang Gung Memorial Hospital.

\section{Animal grouping, time courses of Oxaliplatin and SAHA treatment, and tumor size measurement}

The pathogen-free, 12-week-old male nude mice (Charles River Technology, BioLASCO, Taiwan) were used in this study. We equally separated these mice into four groups. Group 1: DLD-1 cells $\left(2.0 \times 10^{6}\right.$ cells $)$ were subcutaneously injected to the back of nude mice without treating any drugs; Group 2: shCHD4 DLD-1 cells $\left(2.0 \times 10^{6}\right.$ cells $)$ were subcutaneously injected to the back of nude mouse without treating with any drugs; Group 3: DLD-1 cells $\left(2.0 \times 10^{6}\right.$ cells $)$ were subcutaneously injected to the back of nude mice and intra-peritoneal injected with oxaliplatin (12 $\mathrm{mg} / \mathrm{kg} /$ week) from day 14 to 32; Group 4: DLD-1 cells $\left(2.0 \times 10^{6}\right.$ cells $)$ were subcutaneously injected to the back of nude mice and intra-peritoneally injected with oxaliplatin (12 $\mathrm{mg} / \mathrm{kg} /$ week $)$ and suberoylanilide hydroxamic acid (SAHA) (50 $\mathrm{mg} / \mathrm{kg} /$ day) from day 14 to day 32. Tumor growth was measured using the following formula: tumor volume $=\left(\right.$ width $^{2} \times$ length $) / 2$ after the implantation of the tumor cells. Tumors were removed and measured after euthanizing the animals at day 32 post-implantation. Tumor specimens were used for immunohistochemical (IHC) staining and protein extraction. The dosage of oxaliplatin and SAHA was based on previous studies [33, 34] with some modifications.

\section{Human samples}

A total of 40 paired CRC tissue specimens were collected in the Tissue Bank, Kaohsiung Chang Gung Memorial Hospital. All participants provided written informed consent at the time of recruitment. All cases were confirmed with diagnoses of colorectal cancer clinically and pathologically based on the revised international system for staging of colorectal cancer and tissues were kept at $-80^{\circ} \mathrm{C}$ for storage. The Institutional Review Board approval for using these human tissues in this study was given by the Research Ethics Committee of the Kaohsiung Chang Gung Memorial Hospital (IRB-201701900B0) on 25 Nov 2016.

\section{Immunohistochemistry staining and scoring}

Colorectal cancer tissue microarrays (SuperBioChips Laboratories, Gangnam-gu, KR) were 
used in this study. The details on clinicopathological and survival information of patients were provided on the manufacturer's websites. To perform the immunohistochemistry (IHC) staining, paraffin embedded tissue sections were de-paraffinized, rehydrated, retrieval, treated with $\mathrm{H}_{2} \mathrm{O}_{2}$ and then incubated with primary antibodies specifically against CHD4, appropriate secondary antibody and the Envision system (Dako, Denmark) [26]. Finally, sections were counterstained with hematoxylin and then analyzed by microscope. CHD4 expression has been defined as the nucleus staining in tumor cells and CHD4 expression in different patients was scored based on the product of signal intensity and the proportion of positive cells [25, 26, 35].

\section{Statistical analysis}

CHD4 expression determined using IHC staining in CRC tissues was compared with patient survival and assessed using the Chi-square test. To evaluate the significance of CHD4 in CRC prognosis, survival curves were obtained using the KaplanMeier method. In addition, the difference between each group was compared and calculated by two-tailed Student's $t$-test. $P<0.05$ was considered statistically significant. All statistical analyses were performed using SPSS 19.0 software (IBM Corp.,
Armonk, NY, USA).

\section{Results}

\section{Associations between CHD4 and clinicopathological parameters in CRC patients}

CHD4 has been reported as an important regulator in several cancer types. However, its role in $\mathrm{CRC}$ is still unclear. To assess the role of CHD4 in CRC patients, we used colon cancer samples from the Ualcan database by The Cancer Genome Atlas to analyze the association between CHD4 mRNA expression and several clinical parameters. Our results showed that regardless of patient ethnicity (Caucasian, African-American, and Asian), histological subtypes (such as adenocarcinoma and mucinous adenocarcinoma), nodal metastasis (N0 to N2), TP53 mutation status, and individual cancer stages, CRC patients expressed significantly higher CHD4 mRNA expression than healthy individuals (Fig. 1A-E). The Kaplan-Meier plotter database showed that high CHD4 mRNA expression was also correlated with short overall survival (Fig. 1F). Thus, CHD4 plays an important role in regulating the malignant characteristics of CRC.
(A)

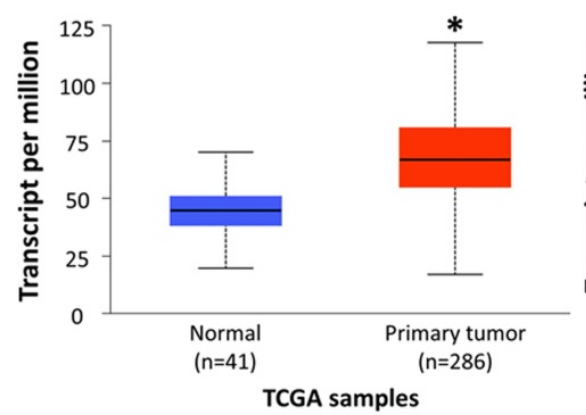

(D)

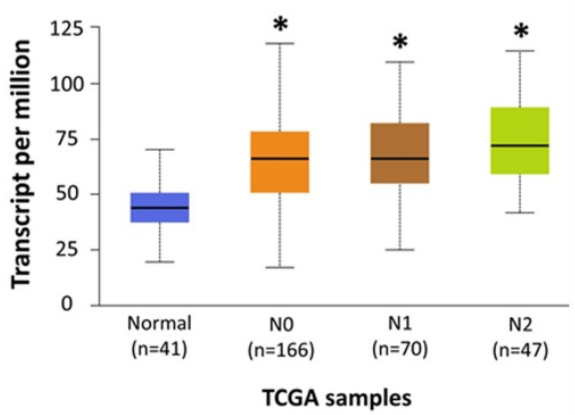

(B)

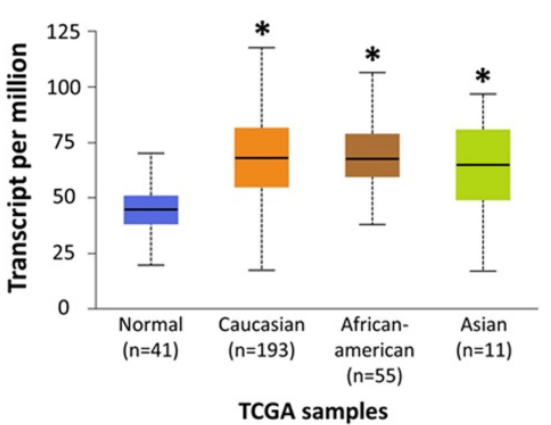

(E)

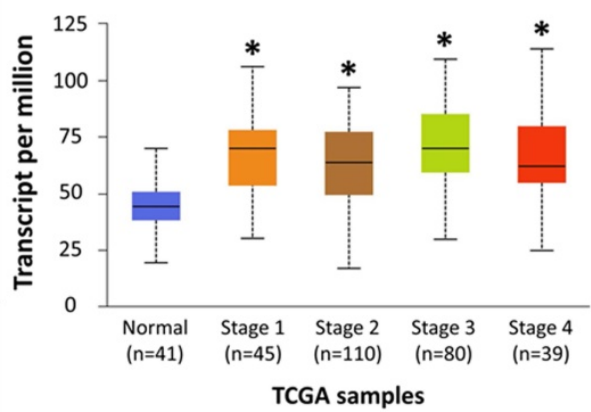

(C)
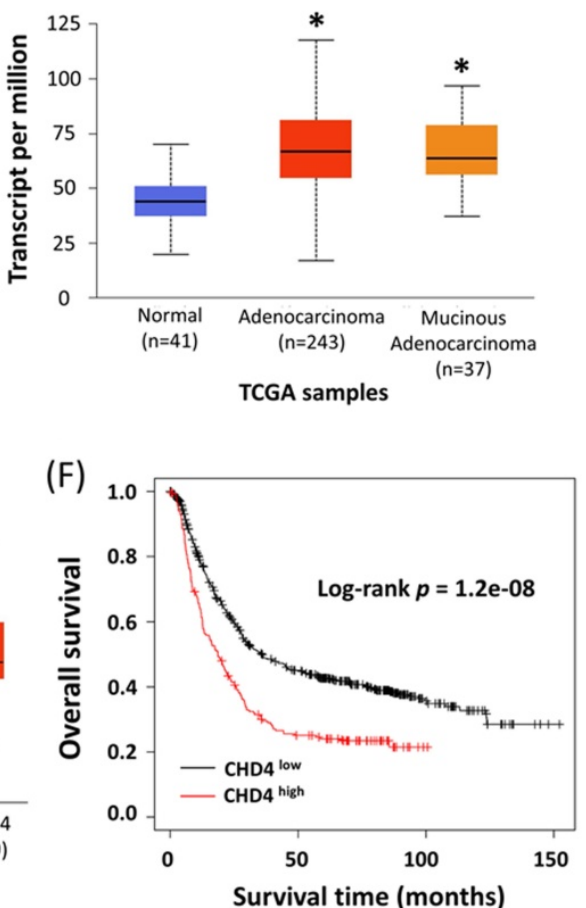

Figure 1. Higher expression of CHD4 mRNA is associated with poor prognosis in CRC patients. (A-E) Patients with CRC express significantly higher CHD4 mRNA than healthy individuals (A). This tendency is observed regardless of patient ethnicity (Caucasian, African-American, and Asian) (B), histological subtypes (such as adenocarcinoma and mucinous adenocarcinoma) (C), nodal metastasis (N0 to N2) (D), and individual cancer stages (E). All patients with CRC express significantly higher level of CHD4 mRNA than healthy individuals. These data are obtained from UALCAN database in TCGA CRC cancer samples. (F) Kaplan-Meier analyses of overall survival in CRC patients from online KM plotter. Patients are stratified into 'low' and 'high' CHD4 mRNA expression based on auto select best cutoff (The highest HR value (or 1/HR in case of HR<1) in case of $P=0$ in the Cox regression). $* P<0.05$. HR: Hazard ratio. 
(A)

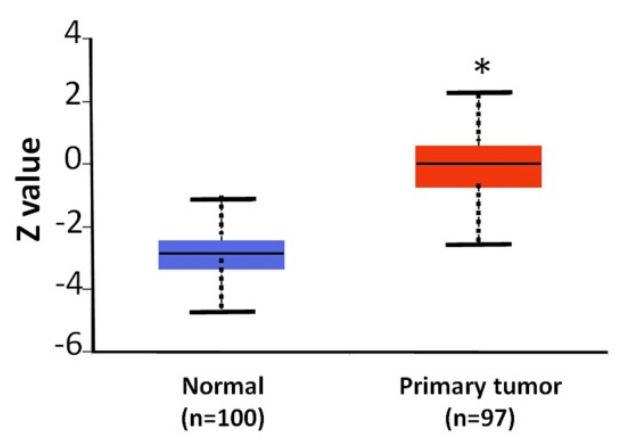

(C)

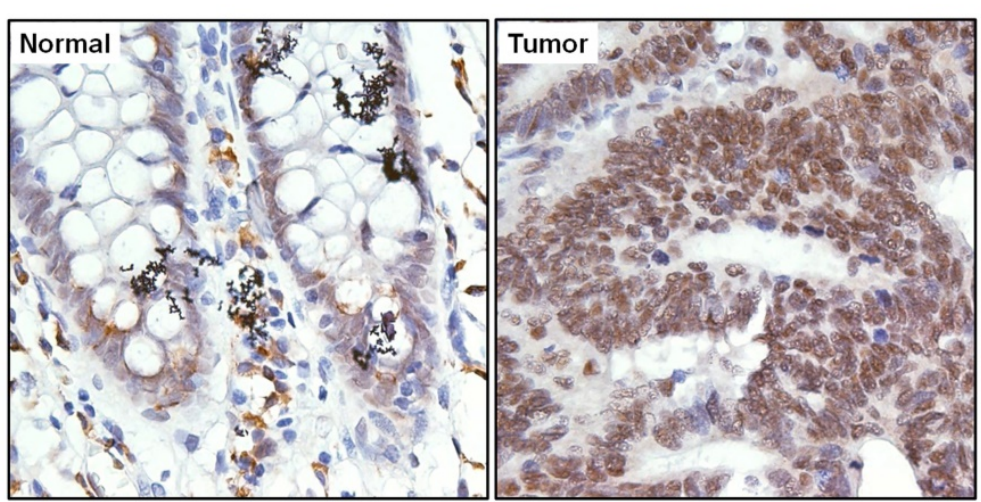

(B)

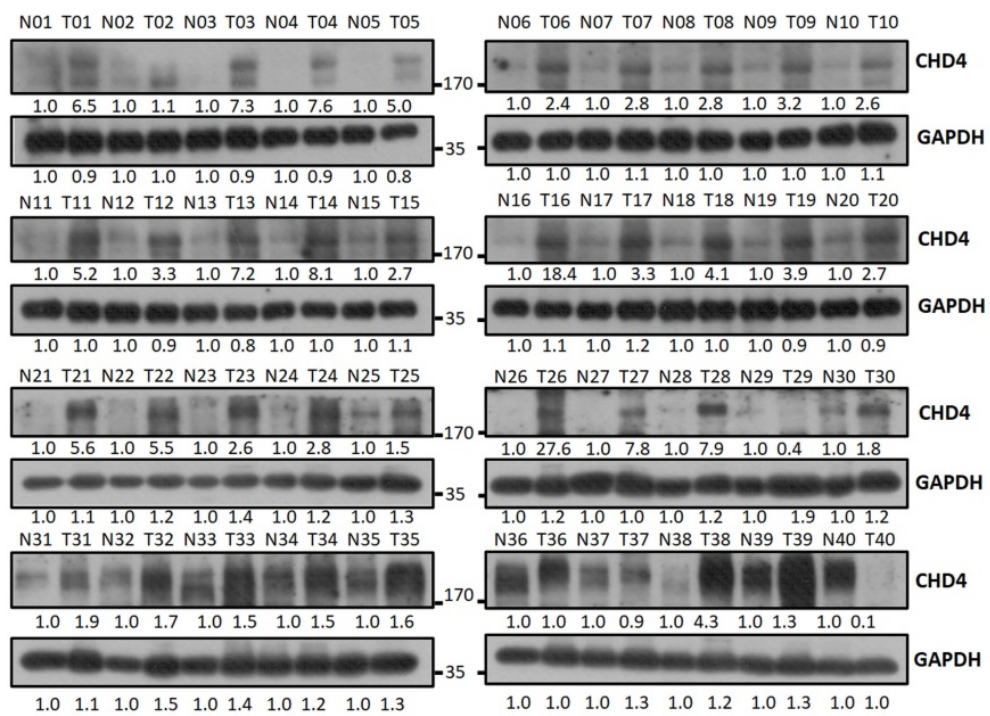

(D)

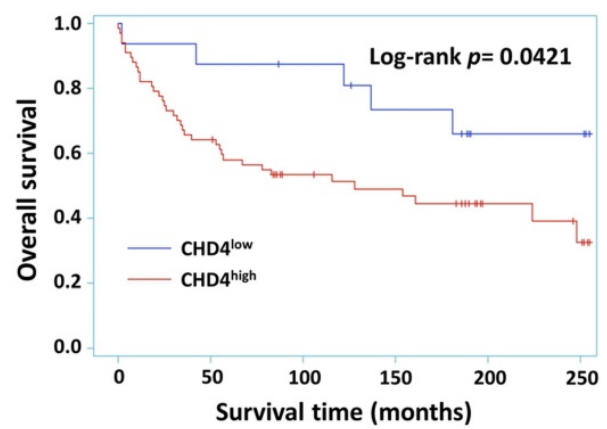

Figure 2. Representative immunohistochemistry staining results and protein expression of CHD4 in tumor cells of CRC patients. (A) Protein expression of CHD4 in normal and tumor tissues analyzed by CPTAC in CRC patients. (B) CHD4 protein expression in 40 CRC patients. (C) CHD4 expression in normal and tumor tissue in CRC patients (original 400x magnification). The classification of CHD4 expression was according to the staining observed in the cell nucleus. (D) Kaplan-Meier survival curves in $C R C$ patients.

\section{CHD4 protein expression is usually overexpressed in most CRC patients and correlates with patient survival}

We have found that the mRNA expression of CHD4 was highly expressed in CRC patients from the Ualcan database. In addition, the result of Clinical Proteomic Tumor Analysis Consortium (CPTAC) analysis also showed that CHD4 protein expression in CRC tissues was also higher than it in normal tissues (Fig. 2A). In this study, we collected 40 CRC patient samples in our hospital and analyzed CHD4 protein expression. As shown in Fig. 2B, most paired samples from the same CRC patients showed that CHD4 protein expression was higher in tumor tissues than in adjacent non-tumor colon epithelial tissues (About $85 \%$ of CHD4 protein expression in tumor tissues were at least 1.5 times higher than adjacent non-tumor colon epithelial tissues), suggested that CHD4 could be a diagnostic marker in CRC cancer. Next, we used the tissue microarrays (TMA) to investigate the relationships between CHD4 and several clinical parameters in CRC patients. Fig. $2 \mathrm{C}$ shows the representative IHC staining results of the nuclear levels of CHD4 in adjacent non-tumor colon epithelial tissue and tumor tissue from CRC patients. CHD4 expression was higher in the nuclei of tumor cells than in normal colon epithelial cells. Table 1 indicates that CHD4 expression was significantly associated with the metastatic stage and survival. Kaplan-Meier analysis also showed that the survival duration in patients with high CHD4 levels was significantly lower than that in patients with low CHD4 expression (Fig. 2D). Thus, CHD4 has the potential to be an important diagnostic and prognostic biomarker in CRC patients.

\section{CHD4 affects CRC cell malignant characteristics such as cell proliferation and motility}

Our clinical analytical results have shown that CHD4 expression is associated with nodal and 
metastasis stage and survival. Several prior studies suggested that CHD4 could regulate several malignant characteristics in cancer cells $[18,21,25,26$, $35,36]$. Therefore, we investigated whether CHD4 affected the proliferation and motility of CRC cells. At first, we found that knockdown of CHD4 using siRNA or shRNA significantly suppressed cell proliferation in DLD-1 cells after 72h culture (Fig. 3A and Fig. S1A). This phenomenon was also seen in other CRC cell lines (Fig. S1B). Next, we found that knockdown of CHD4 using siRNA or shRNA all significantly reduced the number of migrated and invaded in DLD-1 cells (Fig. 3B and C), suggested that CHD4 has effects on regulating cell motility of CRC cells. Our previous study found that CHD4 plays a role in regulating the loss of E-cadherin, one of the epithelial-mesenchymal transition (EMT) protein, then promoting metastatic abilities in TNBC cells [26]. In this study, we also found that several EMT-related proteins (such as Vimentin, MMP2, and N-cadherin) were decreased while that of E-cadherin was increased in CHD4-depleted DLD-1 cells (Fig. 3D). Hence, these results suggest that CHD4 affected the migratory and invasive abilities of CRC cells through altering several proteins associated with EMT in CRC cells.

Table 1. Relationship between CHD4 expression and clinicopathological characteristics of CRC patients $(n=83)$

\begin{tabular}{|c|c|c|c|c|}
\hline \multirow[t]{2}{*}{ Parameters } & \multirow[t]{2}{*}{$\mathrm{n}$} & \multicolumn{2}{|c|}{ CHD4, n (\%) } & \multirow[t]{2}{*}{$P$-value } \\
\hline & & Low & High & \\
\hline Total & 83 & $25(30.12)$ & $58(69.88)$ & \multirow{4}{*}{0.4252} \\
\hline \multicolumn{4}{|l|}{ Tumor stage } & \\
\hline $\mathrm{T} 1 / \mathrm{T} 2$ & 7 & $3(12.00)$ & $4(6.90)$ & \\
\hline $\mathrm{T} 3 / \mathrm{T} 4$ & 76 & $22(88.00)$ & $54(93.10)$ & \\
\hline \multicolumn{4}{|l|}{ Nodal stage } & \multirow[t]{3}{*}{0.2402} \\
\hline No & 45 & $16(64.00)$ & $29(50.00)$ & \\
\hline N1/N2/N3 & 38 & $9(36.00)$ & $29(50.00)$ & \\
\hline \multicolumn{4}{|c|}{ Metastatic stage } & \multirow[t]{3}{*}{$0.0315^{*}$} \\
\hline M0 & 68 & $24(96.00)$ & $44(75.86)$ & \\
\hline M1 & 15 & $1(4.00)$ & $14(24.14)$ & \\
\hline \multicolumn{4}{|l|}{ Survival status } & \multirow[t]{3}{*}{$0.0260^{*}$} \\
\hline Survival & 41 & $17(68.00)$ & $24(41.38)$ & \\
\hline Death & 42 & $8(32.00)$ & 34 (58.62) & \\
\hline
\end{tabular}

*Statistically significant $(\mathrm{P}<0.05)$.
(A)

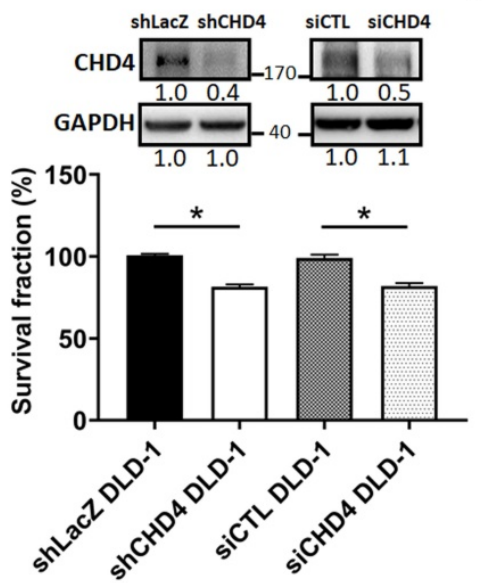

(C)

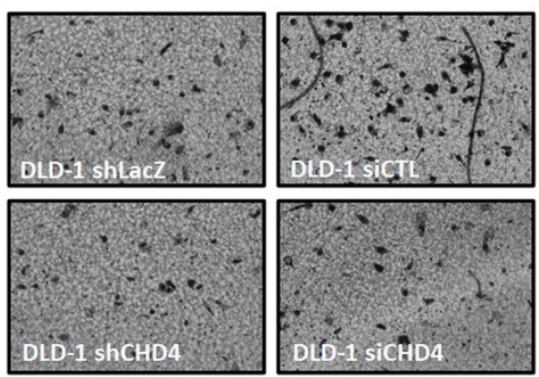

(B)

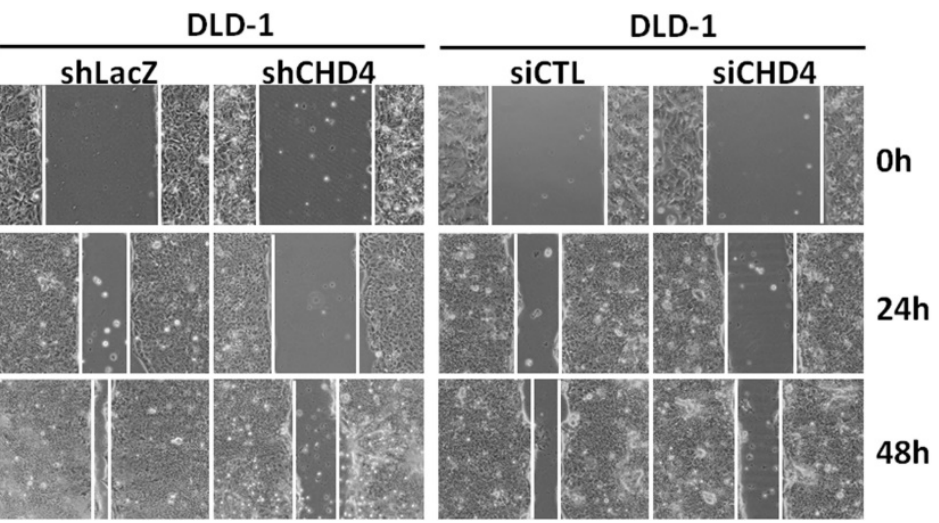

(D)

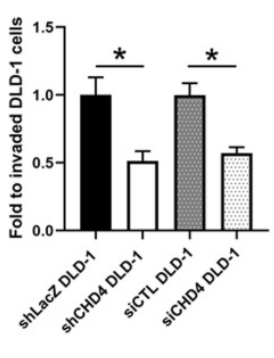

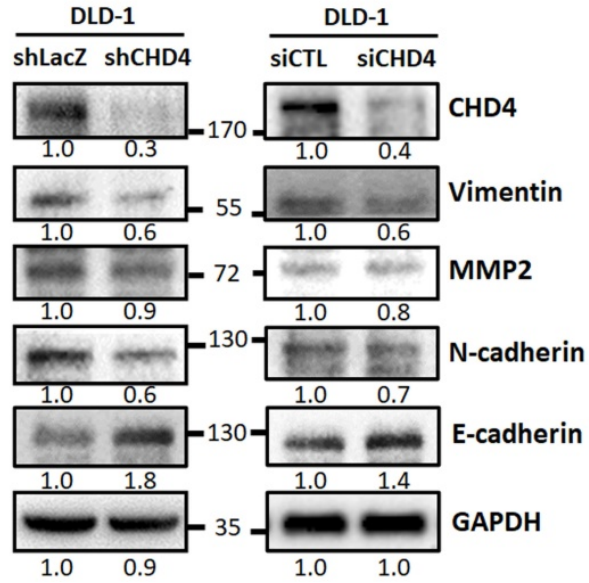

Figure 3. CHD4 mediates cell motility in CRC cells through the epithelial-mesenchymal transition (EMT)-related mechanism. (A) Knockdown of CHD4 in DLD-1 suppresses the cell proliferation. (B) Knockdown of CHD4 suppresses DLD-1 cell migration. (C) Knockdown of CHD4 suppresses DLD-1 cell invasion. (D) Knockdown of CHD4 affects several EMT-associated protein expressions (such as Vimentin, MMP2, N-cadhenin, and E-cadherin) in CRC cells. Data from three independent experiments are used for statistical analysis and $* P<0.05$. 
(A)

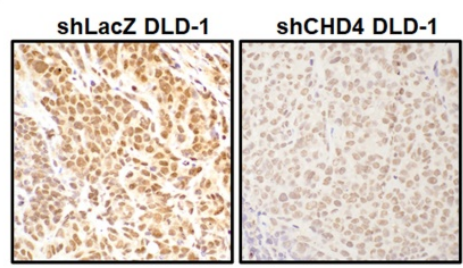

(C)
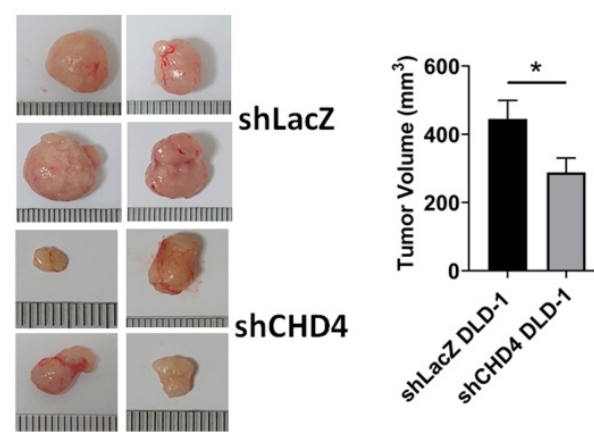

Figure 4. Suppression of CHD4 affects the CRC tumor cell growth and increases sensitivity to cisplatin. (A) IHC staining of CHD4 expression in shLacZ and shCHD4 tumor tissues (original 400x magnification). (B) Knockdown of CHD4 reduces the tumor growth rate. (C) Tumor size in CHD4 depleted DLD-1 tumor is significantly smaller than that in parental DLD-1 tumor. *: $P<0.05$.

(A)

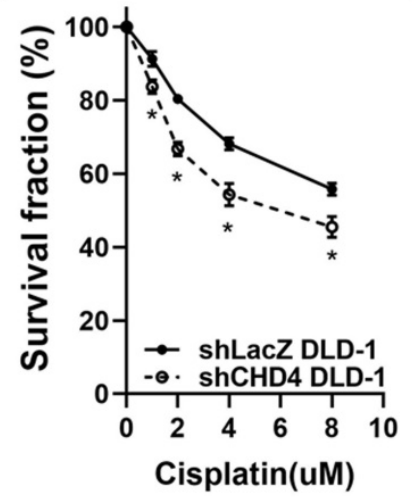

(B)

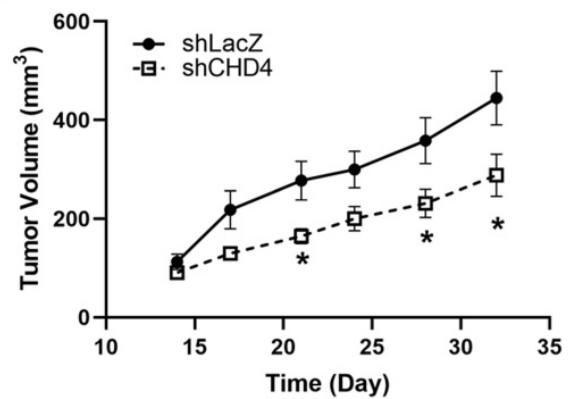

Suppression of CHD4 affects the tumor growth in vivo

After a series of in vitro experiments, we found that CHD4 played important roles in regulating cell proliferation in CRC cells. In order to verify our in vitro findings, we next established a xenograft CRC animal model. shLacZ and shCHD4 DLD-1 cells were implanted subcutaneously into the back of the mice. As shown in Fig. 4A, the IHC staining revealed high $\mathrm{CHD} 4$ expression in shLAcZ and low CHD4 expression in shCHD4 tumor tissues. In addition, the tumor growth in shCHD4 DLD-1 cells was slower than that in shLacZ DLD-1 cells (Fig. 4B) and the tumor size in shCHD4 DLD-1 tumor was also significant smaller than shLacZ DLD-1 tumor (Fig. 4C). Thus, these results demonstrated that CHD4 could mediate the CRC tumor growth in vivo.

\section{Inhibition of CHD4 increases the sensitivity of CRC cells to cisplatin}

Previous studies have indicated that high CHD4 expression was associated with anti-cancer drug and radiation resistance in several different cancer types [25-27, 37-39]. Thus, we investigated the role of CHD4 in regulating the platinum drug sensitivity in CRC cells. As shown in Fig. 5A, knockdown of CHD4 in DLD-1 cells increased the cytotoxicity induced by cisplatin. Other CRC cancer cells, such as HCT-116 and HT-29, also exhibited the similar sensitizing effect by cisplatin in CHD4 knockdown cells (Fig. S1C). Moreover, the results of the flow cytometry and western blot analysis also showed that cisplatin could induce higher rate of apoptosis in shCHD4 DLD-1 cells (Fig. 5B and C). In addition, knockdown CHD4 by siRNA also resulted in similar results (Fig. S1D

Figure 5. CHD4 plays a role in regulating drug sensitivity in CRC cells. (A) Knockdown of CHD4 in DLD-1 can increase the cytotoxicity of cisplatin. (B) AnV-PI double staining shows that cisplatin induces higher cell death rate through apoptosis in CHD4 depleted DLD-1 cells. (C) Western blot analysis shows that higher levels of cleaved Caspase3 and PARP in CHD4 depleted DLD-1 cells were treated with cisplatin. Data from three independent experiments are used for statistical analysis and $* P<0.05$. 
to F). Therefore, our results indicated that defects in CHD4 resulted in greater platinum druginduced toxicity in CRC cells, supposed that CRC patients who expressed low CHD4 are more suitable for platinum drug treatment.

\section{Destabilized NuRD complex can increase sensitivity to platinum drugs in CRC cells}

Although CHD4 plays important in regulating cell proliferation, motility, platinum drug sensitivity in CRC cells, however, there is no significant inhibitor developed for CHD4 until now. It is well known that CHD4 and HDAC1 are associated with the NuRD complex [15]. Thus, we used vorinostat (SAHA), an HDAC inhibitor (HDACi), to destabilize the NuRD complex and to test the role of CHD4 in regulating anticancer drug sensitivity. Surprisingly, our in vitro results showed that SAHA could suppress CHD4 expression in DLD-1 cells (Fig. 6A) and other CRC cells (Fig. S1G). MTT assay also showed that the combination of cisplatin and SAHA could also increase the cytotoxicity of cisplatin, and this sensitizing effect is similar to the depletion of $\mathrm{CHD} 4$ then combining with cisplatin (Fig. 6B). In addition, this combination treatment also induced more apoptotic cell death than SAHA or cisplatin treatment alone (Fig. 6C). Our in vivo data showed that this combination also inhibited the tumor growth (Fig. 6D) and immunoblotting also showed that the expression of apoptotic markers, such as the cleavages of PARP and Caspase 3, as well as DNA damage markers $(\gamma-\mathrm{H} 2 \mathrm{AX})$ were increased when combining oxaliplatin and SAHA (Fig. 6E). These results suggested that this combination regulated drug sensitivity could through destabilizing the NuRD complex. Based on our results, this combination treatment can be applied for CRC patients who have high CHD4 expression. Moreover, the impairment of the NuRD complex can increase the sensitivity to oxaliplatin in CRC cells.

\section{Discussion}

CRC is the third most common cancer in developed countries. Previous studies have indicated that genomic instability, such as chromosomal and microsatellite instabilities, played pivotal roles in CRC development [40]. In current clinical practice, surgical resection is the major choice for CRC treatment. Chemo- and radio-therapies are preoperative or postoperative adjuvant therapies for patients with CRC. Although $70-80 \%$ CRC tumors can be removed
(A)

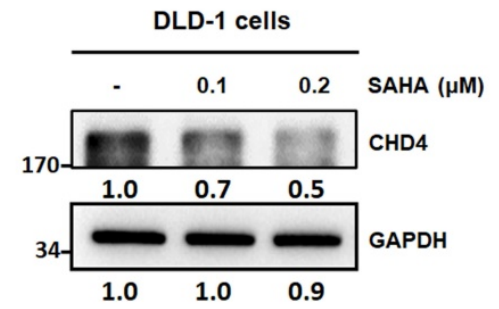

(B)

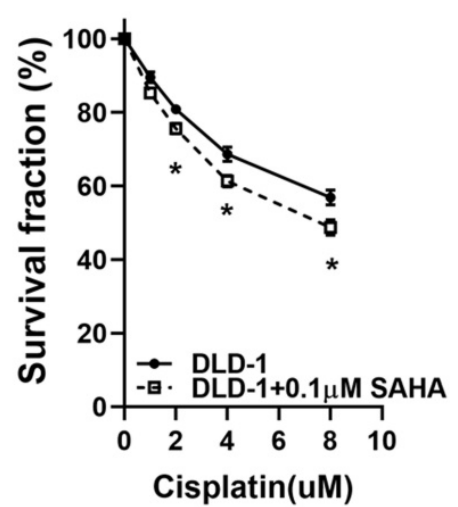

(D)
(C)
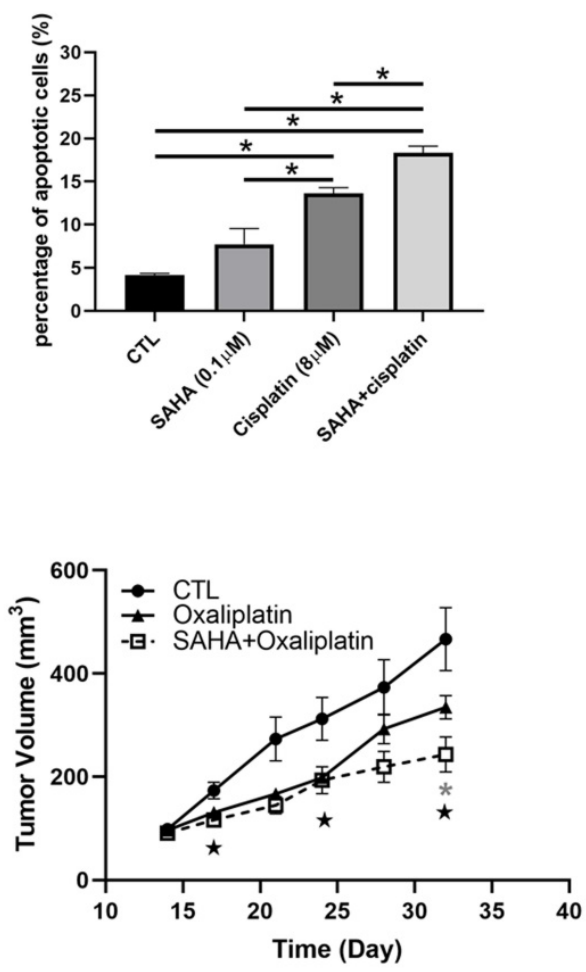

(E)

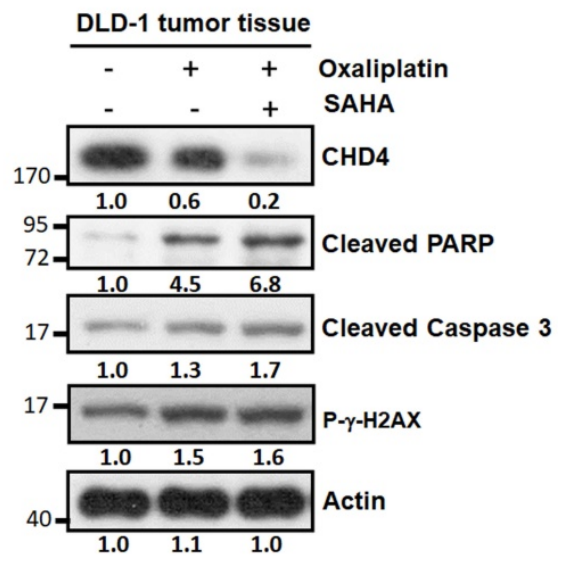

Figure 6. Impaired of NuRD complex can increase platinum drug sensitivity in CRC cells. (A) SAHA shows the potential in suppressing CHD4 expression in DLD-1 cells. (B) The combination of cisplatin and SAHA increases the cisplatin cytotoxicity in DLD-1 cells. (C) The combination of cisplatin and SAHA treatment induces more apoptotic cell death than SAHA or cisplatin treatment alone. (D) The combination of oxaliplatin and SAHA inhibits the DLD-1 tumor growth in animal model $\star$ : P $<0.05$ in CTL group vs. SAHA+ Oxaliplatin group; $*$ : $<<0.05$ in Oxaliplatin group vs. SAHA+ Oxaliplatin group. (E) Western blot analysis shows that the combination of oxaliplatin and SAHA can induce higher rate of apoptotic cell death in DLD-1 tumor model. Data from three independent experiments were used for statistical analysis and *: $P<0.05$. 
by surgical resection, about half of the patients still exhibit poor response and are resistant to treatments. This results in recurrence and distal metastasis [41, 42]. In the past two decades, several investigators have indicated that CRC was a heterogeneous and multistep disease caused by genetic and epigenetic aberrations under microenvironmental influence [13]. They also suggested that epigenetic regulation was an important event in colorectal carcinogenesis in addition to genetic alterations.

Several epigenetic mechanisms, such as DNA and histone modification, result in the inheritable silencing of genes without a change in their coding sequence. Epigenetic modulators have become increasingly relevant in clinical practice, especially in applying as diagnostic and prognostic markers as well as novel therapeutic target development [43]. Previous studies also indicated that NuRD-mediated chromatin remodeling was necessary for further deposition of epigenetic repressive marks. It was also involved in the progression to advanced stages in different types of cancer [44-48]. Among the components of the NuRD complex, CHD4 plays an oncogenic role that initiates and supports TSG silencing in various cancer types $[18,19,21-24,26,27$, $36,39]$.

In the context of DNA damage, CHD4 is associated with abnormal promoter $\mathrm{CpG}$ island DNA methylation and has a key role in initiating and maintaining epigenetic gene silencing [49]. This role has been shown to be integral to the oncogenic properties of CHD4 [21, 23, 45]. High expression of CHD4 has been found to be associated with poor prognosis in various cancer types [18, 21, 23-27, 36]. Recent studies also indicated that CHD4 recruited repressive chromatin proteins to help maintain DNA hypermethylation-associated transcriptional silencing of TSGs. High expression of CHD4 in CRC patients was also correlated with radio-resistance [25]. In addition, recent studies suggested that $\mathrm{CHD} 4$ has a potential to be a prognostic biomarker in triple-negative breast cancer [26, 35] and high CHD4 and 8-hydroxy-deoxyguanosine levels as well as low expression of TSGs were strongly correlated with early disease recurrence and decreased overall survival in CRC patients [21].

In this study, we evaluated the role of CHD4 in regulating several malignant characteristics, such as cell proliferation, motility, drug sensitivity, and tumor growth, through clinical databases, clinical samples, in vitro cell models, and in vivo animal models. Our results indicated that most CRC patients had higher CHD4 expression in tumor tissues than in normal tissues. Moreover, higher CHD4 expression was correlated with nodal and distant metastasis as well as poorer survival rates. CHD4 also affected cell proliferation and motility by regulating several EMT-related proteins. Knockdown of CHD4 expression might suppress the abovementioned phenomena and increase the platinum drug sensitivity. In animal model, we found that tumor growth in CHD4-depleted cells was lower than that in parental cells, suggesting that high expression of CHD4 promoted tumor growth in CRC.

Although there were no specific inhibitors for CHD4, we used SAHA, one of the HDAC inhibitors, which has potential to destabilize the NuRD complex, in this study. To verify whether using HDAC inhibitors was a viable alternative treatment strategy for CRC patients with high CHD4 expression, SAHA was used in combination with platinum drugs to treat DLD-1 cells and DLD-1 tumors. Our data showed that SAHA inhibited CHD4 expression in CRC cells. Additionally, the combination of SAHA and platinum drugs also suppressed CHD4 protein expression in vitro and in vivo. This combination also increased the cytotoxicity of platinum drug alone. Previous studies also associated the synergism between HDACi and oxaliplatin with the augmented apoptotic signal. This allowed for the significant dose reduction of anticancer agents [50]. However, the mechanisms of downregulation of CHD4 expression by SAHA should be investigated in the further study. Therefore, CHD4 is a potential biomarker for selecting patients to be treated with the combination of HDACis and platinum-drugs. In addition, it is necessary to develop CHD4 inhibitors for CRC patients in the future.

\section{Conclusions}

In summary, we found that CHD4 was overexpressed in tumor tissues in CRC patients. High expression of CHD4 was correlated with poor survival as well as high nodal and distant metastases. In addition, CHD4 regulated tumor growth rate and drug sensitivity. Thus, CHD4 is a potential drug target for treating patients with CRC.

\section{Supplementary Material}

Supplementary figure $\mathrm{S1}$.

http://www.ijbs.com/v17p1660s1.pdf

\section{Acknowledgements}

\section{Funding}

This work was supported by the following grants: (1) Chang Gung Memorial Hospital Research Project, Taiwan (CMRPG8H1291). (2) 109-2314-B037-036-MY3 from the Ministry of Science and Technology, Taiwan. (3) KMUH109-9R36 from the Kaohsiung Medical University Hospital, Taiwan. We 
also thank the National RNAi Core Facility at Academia Sinica in Taiwan for providing shRNA reagents and related services.

\section{Author Contributions}

Investigation, Chia-Lo Chang, Chi-Ruei Huang and Hong-Hwa Chen; Methodology, Chia-Lo Chang, Chi-Ruei Huang and Hong-Hwa Chen; Revised experiment performing, Shu-Jyuan Chang and Chun-Chieh $\mathrm{Wu}$; Supervision, Chi-Wen Luo and Hon-Kan Yip; Writing - review \& editing, Chi-Wen Luo and Hon-Kan Yip.

\section{Competing Interests}

The authors have declared that no competing interest exists.

\section{References}

1. Arnold M, Sierra MS, Laversanne M, Soerjomataram I, Jemal A, Bray F. Global patterns and trends in colorectal cancer incidence and mortality. Gut. 2017; 66: 683-91.

2. Markowitz SD, Bertagnolli MM. Molecular origins of cancer: Molecular basis of colorectal cancer. N Engl J Med. 2009; 361: 2449-60.

3. Vaiopoulos AG, Athanasoula K, Papavassiliou AG. Epigenetic modifications in colorectal cancer: molecular insights and therapeutic challenges. Biochim Biophys Acta. 2014; 1842: 971-80.

4. Oshima H, Oshima M. The inflammatory network in the gastrointestinal tumor microenvironment: lessons from mouse models. J Gastroenterol. 2012; 47: 97-106.

5. Rustgi AK. The genetics of hereditary colon cancer. Genes Dev. 2007; 21: 2525-38.

6. Abu-Remaileh M, Bender S, Raddatz G, Ansari I, Cohen D, Gutekunst J, et al. Chronic inflammation induces a novel epigenetic program that is conserved in intestinal adenomas and in colorectal cancer. Cancer Res. 2015; 75: 2120-30

7. Li X, Pu J, Jiang S, Su J, Kong L, Mao B, et al. Henryin, an ent-kaurane diterpenoid, inhibits Wnt signaling through interference with beta-catenin/TCF4 interaction in colorectal cancer cells. PLoS One. 2013; 8: e68525.

8. Migheli F, Migliore L. Epigenetics of colorectal cancer. Clin Genet. 2012; 81: $312-8$.

9. van Engeland M, Derks S, Smits KM, Meijer GA, Herman JG. Colorectal cancer epigenetics: complex simplicity. J Clin Oncol. 2011; 29: 1382-91.

10. Lao VV, Grady WM. Epigenetics and colorectal cancer. Nat Rev Gastroenterol Hepatol. 2011; 8: 686-700.

11. Goel A, Xicola RM, Nguyen TP, Doyle BJ, Sohn VR, Bandipalliam P, et al. Aberrant DNA methylation in hereditary nonpolyposis colorectal cancer without mismatch repair deficiency. Gastroenterology. 2010; 138: 1854-62.

12. Kondo Y, Issa JP. Epigenetic changes in colorectal cancer. Cancer Metastasis Rev. 2004; 23: 29-39.

13. Bardhan K, Liu K. Epigenetics and colorectal cancer pathogenesis. Cancers (Basel). 2013; 5: 676-713.

14. Zarkavelis G, Boussios S, Papadaki A, Katsanos KH, Christodoulou DK, Pentheroudakis G. Current and future biomarkers in colorectal cancer. Ann Gastroenterol. 2017; 30: 613-21.

15. Basta J, Rauchman M. The nucleosome remodeling and deacetylase complex in development and disease. Transl Res. 2015; 165: 36-47.

16. Zhang Y, LeRoy G, Seelig HP, Lane WS, Reinberg D. The dermatomyositis-specific autoantigen $\mathrm{Mi} 2$ is a component of a complex containing histone deacetylase and nucleosome remodeling activities. Cell. 1998; 95: 279-89.

17. Xue Y, Wong J, Moreno GT, Young MK, Cote J, Wang W. NURD, a novel complex with both ATP-dependent chromatin-remodeling and histone deacetylase activities. Mol Cell. 1998; 2: 851-61.

18. D'Alesio C, Punzi S, Cicalese A, Fornasari L, Furia L, Riva L, et al. RNAi screens identify CHD4 as an essential gene in breast cancer growth. Oncotarget. 2016; 7: 80901-15.

19. O'Shaughnessy A, Hendrich B. CHD4 in the DNA-damage response and cell cycle progression: not so NuRDy now. Biochem Soc Trans. 2013; 41: 777-82.
20. Polo SE, Kaidi A, Baskcomb L, Galanty Y, Jackson SP. Regulation of DNA-damage responses and cell-cycle progression by the chromatin remodelling factor CHD4. EMBO J. 2010; 29: 3130-9.

21. Xia L, Huang W, Bellani M, Seidman MM, Wu K, Fan D, et al. CHD4 Has Oncogenic Functions in Initiating and Maintaining Epigenetic Suppression of Multiple Tumor Suppressor Genes. Cancer Cell. 2017; 31: 653-68 e7.

22. Pan MR, Hsieh HJ, Dai H, Hung WC, Li K, Peng G, et al. Chromodomain helicase DNA-binding protein 4 (CHD4) regulates homologous recombination DNA repair, and its deficiency sensitizes cells to poly(ADP-ribose) polymerase (PARP) inhibitor treatment. J Biol Chem. 2012; 287: 6764-72.

23. Sperlazza J, Rahmani M, Beckta J, Aust M, Hawkins E, Wang SZ, et al. Depletion of the chromatin remodeler CHD4 sensitizes AML blasts to genotoxic agents and reduces tumor formation. Blood. 2015; 126: 1462-72.

24. D'Alesio C, Bellese G, Gagliani MC, Lechiara A, Dameri M, Grasselli E, et al. The chromodomain helicase CHD4 regulates ERBB2 signaling pathway and autophagy in ERBB2(+) breast cancer cells. Biol Open. 2019; 8 .

25. Wang HC, Chou CL, Yang CC, Huang WL, Hsu YC, Luo CW, et al. Over-Expression of CHD4 Is an Independent Biomarker of Poor Prognosis in Patients with Rectal Cancers Receiving Concurrent Chemoradiotherapy. Int J Mol Sci. 2019; 20.

26. Luo CW, Wu CC, Chang SJ, Chang TM, Chen TY, Chai CY, et al. CHD4-mediated loss of E-cadherin determines metastatic ability in triple-negative breast cancer cells. Exp Cell Res. 2018; 363: 65-72.

27. Hou MF, Luo CW, Chang TM, Hung WC, Chen TY, Tsai YL, et al. The NuRD complex-mediated p21 suppression facilitates chemoresistance in BRCA-proficient breast cancer. Exp Cell Res. 2017; 359: 458-65.

28. Cai Y, Geutjes EJ, de Lint K, Roepman P, Bruurs L, Yu LR, et al. The NuRD complex cooperates with DNMTs to maintain silencing of key colorectal tumor suppressor genes. Oncogene. 2014; 33: 2157-68.

29. Geeleher P, Loboda A, Lenkala D, Wang F, LaCroix B, Karovic S, et al. Predicting Response to Histone Deacetylase Inhibitors Using High-Throughput Genomics. J Natl Cancer Inst. 2015; 107.

30. Luo $\mathrm{CW}, \mathrm{Wu} \mathrm{CC}, \mathrm{Ch}$ 'ang $\mathrm{HJ}$. Radiation sensitization of tumor cells induced by shear stress: the roles of integrins and FAK. Biochim Biophys Acta. 2014; 1843: 2129-37.

31. Sung PH, Luo CW, Chiang JY, Yip HK. The combination of G9a histone methyltransferase inhibitors with erythropoietin protects heart against damage from acute myocardial infarction. Am J Transl Res. 2020; 12: 3255-71.

32. Pan MR, Hou MF, Ou-Yang F, Wu CC, Chang SJ, Hung WC, et al. FAK is Required for Tumor Metastasis-Related Fluid Microenvironment in Triple-Negative Breast Cancer. J Clin Med. 2019; 8.

33. Wang LT, Liou JP, Li YH, Liu YM, Pan SL, Teng CM. A novel class I HDAC inhibitor, MPT0G030, induces cell apoptosis and differentiation in human colorectal cancer cells via HDAC1/PKCdelta and E-cadherin. Oncotarget. 2014; 5: 5651-62.

34. Prewett M, Deevi DS, Bassi R, Fan F, Ellis LM, Hicklin DJ, et al. Tumors established with cell lines selected for oxaliplatin resistance respond to oxaliplatin if combined with cetuximab. Clin Cancer Res. 2007; 13: 7432-40.

35. Ou-Yang F, Pan MR, Chang SJ, Wu CC, Fang SY, Li CL, et al. Identification of CHD4-beta1 integrin axis as a prognostic marker in triple-negative breast cancer using next-generation sequencing and bioinformatics. Life Sci. 2019; 238: 116963.

36. Xu N, Liu F, Wu S, Ye M, Ge H, Zhang M, et al. CHD4 mediates proliferation and migration of non-small cell lung cancer via the RhoA/ROCK pathway by regulating PHF5A. BMC Cancer. 2020; 20: 262.

37. Heshmati Y, Turkoz G, Harisankar A, Kharazi S, Bostrom J, Dolatabadi EK, et al. The chromatin-remodeling factor CHD4 is required for maintenance of childhood acute myeloid leukemia. Haematologica. 2018; 103: 1169-81.

38. Guillemette S, Serra RW, Peng M, Hayes JA, Konstantinopoulos PA, Green MR, et al. Resistance to therapy in BRCA2 mutant cells due to loss of the nucleosome remodeling factor CHD4. Genes Dev. 2015; 29: 489-94.

39. McKenzie LD, LeClair JW, Miller KN, Strong AD, Chan HL, Oates EL, et al. CHD4 regulates the DNA damage response and RAD51 expression in glioblastoma. Sci Rep. 2019; 9: 4444.

40. Nojadeh JN, Behrouz Sharif S, Sakhinia E. Microsatellite instability in colorectal cancer. EXCLI J. 2018; 17: 159-68.

41. Crea F, Nobili S, Paolicchi E, Perrone G, Napoli C, Landini I, et al. Epigenetics and chemoresistance in colorectal cancer: an opportunity for treatment tailoring and novel therapeutic strategies. Drug Resist Updat. 2011; 14: 280-96.

42. Jemal A, Siegel R, Ward E, Hao Y, Xu J, Murray T, et al. Cancer statistics, 2008. CA Cancer J Clin. 2008; 58: 71-96. 
43. Kaminska K, Nalejska E, Kubiak M, Wojtysiak J, Zolna L, Kowalewski J, et al. Prognostic and Predictive Epigenetic Biomarkers in Oncology. Mol Diagn Ther. 2019; 23: 83-95.

44. Lai AY, Wade PA. Cancer biology and NuRD: a multifaceted chromatin remodelling complex. Nat Rev Cancer. 2011; 11: 588-96.

45. Fu J, Qin L, He T, Qin J, Hong J, Wong J, et al. The TWIST/Mi2/NuRD protein complex and its essential role in cancer metastasis. Cell Res. 2011; 21: 275-89.

46. Toh Y, Nicolson GL. The role of the MTA family and their encoded proteins in human cancers: molecular functions and clinical implications. Clin Exp Metastasis. 2009; 26: 215-27.

47. Zhang H, Stephens LC, Kumar R. Metastasis tumor antigen family proteins during breast cancer progression and metastasis in a reliable mouse model for human breast cancer. Clin Cancer Res. 2006; 12: 1479-86.

48. Morey L, Brenner C, Fazi F, Villa R, Gutierrez A, Buschbeck M, et al. MBD3, a component of the NuRD complex, facilitates chromatin alteration and deposition of epigenetic marks. Mol Cell Biol. 2008; 28: 5912-23.

49. Shen H, Laird PW. Interplay between the cancer genome and epigenome. Cell. 2013; 153: 38-55.

50. Flis S, Gnyszka A, Splawinski J. HDAC inhibitors, MS275 and SBHA, enhances cytotoxicity induced by oxaliplatin in the colorectal cancer cell lines. Biochem Biophys Res Commun. 2009; 387: 336-41. 